\section{Can clinical scoring systems improve the diagnostic accuracy in patients with suspected adult appendicitis and equivocal preoperative computed tomography findings?}

\author{
Min Seok Chae ${ }^{1}$, Chong Kun Hong ${ }^{1}$, Young Rock $\mathrm{Ha}^{1}$, \\ Minjung Kathy Chae' ${ }^{2}$, Young Sik Kim ${ }^{1}$, Tae Yong Shin' ${ }^{1}$ Jung Hwan Ahn ${ }^{2}$ \\ 'Department of Emergency Medicine, Daejin Medical Center, Bundang Jesaeng General Hospital, \\ Seongnam, Korea \\ ${ }^{2}$ Department of Emergency Medicine, Ajou University School of Medicine, Suwon, Korea
}

Objective Adult appendicitis (AA) with equivocal computed tomography (CT) findings remains a diagnostic challenge for physicians. Herein we evaluated the diagnostic performance of several clinical scoring systems in adult patients with suspected appendicitis and equivocal CT findings.

Methods We retrospectively evaluated 189 adult patients with equivocal CT findings. Alvarado, Eskelinen, appendicitis inflammatory response, Raja Isteri Pengiran Anak Saleha Appendicitis (RIPASA), and adult appendicitis score (AAS) scores were evaluated, receiver operating characteristic analysis was conducted, and the optimal, low, and high cut-off values were determined for patient classification into three groups: low, intermediate, or high.

Results In total, 61 patients were included in the appendicitis group and 128 in the non-appendicitis group. There were no significant differences between the area under the curve of the clinical scoring systems in the final diagnosis of AA for equivocal appendicitis on CT (Alvarado, 0.698; Eskelinen, 0.710; appendicitis inflammatory response, 0.668; RIPASA, 0.653; AAS, 0.726). A RIPASA score greater than 7.5 had a high positive predictive value (90.9) and an AAS score less than or equal to 5 had a high negative predictive value (91.7) in the diagnosis of AA.

Conclusion The accuracy of clinical scoring systems in the diagnosis of AA with equivocal CT findings was moderate. Therefore, a high RIPASA score may assist in the diagnosis of AA in patients with equivocal CT findings, and a low AAS score may be used as a criterion for patient discharge. Most patients presented with intermediate scores. The patients with equivocal CT findings may be considered as a third diagnostic category of AA.

Keywords Appendicitis; Clinical decision-making; Diagnostic tests, routine; Multidetector computed tomography
eISSN: 2383-4625

Received: 27 May 2017

Revised: 7 August 2017

Accepted: 21 August 2017

Correspondence to: Jung Hwan Ahn Department of Emergency Medicine, Ajou University School of Medicine, 206 World cup-ro, Yeongtong-gu, Suwon 16499, Korea E-mail:erdrajh@naver.com

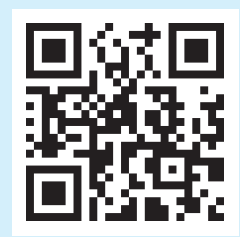

How to cite this article:

Chae MS, Hong CK, Ha YR, Chae MK, Kim YS, Shin TY, Ahn JH. Can clinical scoring systems improve the diagnostic accuracy in patients with suspected adult appendicitis and equivocal preoperative computed tomography findings? Clin Exp Emerg Med 2017;4(4):214-221.
This is an Open Access article distributed under the terms of the Creative Commons Attribution Non-Commercial License (http:// creativecommons.org/licenses/by-nc/4.0/). 


Capsule
What is already known
Computed tomography (CT) scans are widely used in cases of suspected adult appendicitis (AA) due to their accuracy;
however, the diagnosis of AA can still be missed, especially when CT findings are equivocal. The incidence of equivocal
CT findings of AA has been reported to be $5 \%$ to $13.1 \%$, and AA is present in up to 30\% of patients whose CT findings
are considered equivocal. As surgical decisions are made by clinicians based on clinical presentations, AA with equivo-
cal CT findings remain a diagnostic challenge for surgeons and emergency physicians.
What is new in the current study
This is the first study to evaluate the diagnostic power of clinical scoring systems (Alvardo, Eskelinen, appendicitis in-
flammatory response, Raja Isteri Pengiran Anak Saleha Appendicitis [RIPASA] and acute appendicitis score [AAS]) in
patients with suspected AA and equivocal preoperative CT findings. The clinical scoring systems showed overall moder-
ate prognostic performances. A high RIPASA was considered to better diagnose AA in patients with equivocal CT find-
ings and a low AAS was useful to safely discharge patients from the emergency department.

\section{INTRODUCTION}

Adult appendicitis (AA) is a common surgical condition that requires a prompt diagnosis for the minimization of morbidity, mortality, and unnecessary surgical interventions. ${ }^{1}$ The diagnosis of AA using clinical presentation and physical examination is often limited because clinical presentation is atypical and often overlaps with other conditions. ${ }^{1}$ Therefore, the diagnosis of AA needs to be supported by clinical scoring systems or imaging modalities such as ultrasonography and computed tomography (CT).

$\mathrm{CT}$ is widely used in cases of suspected AA because of its accuracy. ${ }^{2-5}$ However, the diagnosis of AA is limited, particularly when $\mathrm{CT}$ findings are equivocal. ${ }^{6}$ The incidence of equivocal CT findings of $A A$ varies between $5.0 \%$ and $13.1 \%{ }^{7}$ and $A A$ occurs in up to $30 \%$ of patients whose CT findings are considered equivocal. ${ }^{6}$ Daly et al. ${ }^{8}$ suggested that equivocal CT results be considered as a diagnostic category and that clinicians should keep in mind that symptomatic patients with equivocal CT scans will have an approximately $30 \%$ chance of having appendicitis. AA with equivocal CT findings remains a diagnostic challenge for clinicians.

In this context, clinical scoring systems may be effectively used to assist in the diagnosis of AA because they are simple and easy to use in clinical practice. To the best of our knowledge, no previous studies have evaluated the diagnostic performance of clinical scoring systems in patients with suspected $\mathrm{AA}$ and equivocal preoperative CT findings. Therefore, we aimed to evaluate the diagnostic performance of various clinical scoring systems in adult patients with suspected $A A$ and equivocal preoperative $C T$ findings. Alvarado score, Eskelinen score, appendicitis inflammatory response (AIR), adult appendicitis score (AAS), and Raja Isteri Pengiran Anak Saleha Appendicitis (RIPASA) scores were analyzed in this study. ${ }^{9-13}$

\section{METHODS}

This study was approved by the institutional review board of Bundang Jesaeng General Hospital, Seongnam, Korea (protocol no. EMC 16-01), and informed consent was waived because the study was based on the retrospective analysis of data.

\section{Study population and data collection}

This single-center, retrospective, observational study evaluated patients that visited the emergency department between April 2011 and November 2013. We collected data on patients older than 15 years with suspected appendicitis and CT evaluation. The patients with equivocal appendicitis on their CT report were enrolled in this study. Patients with equivocal CT findings were divided into two groups according to the final diagnosis: appendicitis group and the non-appendicitis group. The final diagnosis was established via analysis of data from medical records, histological examination, and clinical follow-up collected for 2 weeks after the day of consultation. The final diagnosis of AA was based on the transmural infiltration of neutrophils in the appendix on histological examination. Negative AA was defined as the absence of the histologic findings above among the patients who underwent surgery, or absence of the need for surgical intervention within 2 weeks after the day of consultation in the patients that were not referred to immediate surgery.

We retrospectively collected patient demographics, history, and parameters of the scoring systems from the medical records and the laboratory report system (Table 1). All medical records were carefully reviewed by an emergency physician.

\section{Imaging methods}

All CT examinations were performed using a 16-slice multidetector CT scanner (Brilliance 16, Philips Healthcare, Eindhoven, 
Table 1. Parameters and scores in each clinical scoring system

\begin{tabular}{|c|c|c|c|c|c|}
\hline \multirow{2}{*}{ Parameter } & \multicolumn{5}{|c|}{ Proposed score } \\
\hline & Alvarado & Eskelinen & AIR & RIPASA & AAS \\
\hline \multicolumn{6}{|l|}{ Demographics } \\
\hline Male & - & - & - & 1.0 & - \\
\hline Female & - & - & - & 0.5 & - \\
\hline \multicolumn{6}{|l|}{ Age (yr) } \\
\hline$<40$ & - & - & - & 1.0 & - \\
\hline$\geq 40$ & - & - & - & 0.5 & - \\
\hline Foreign national registration ID card & - & - & - & 1.0 & - \\
\hline \multicolumn{6}{|l|}{ Symptoms } \\
\hline Nausea/vomiting & 1 & - & - & 1.0 & - \\
\hline Vomiting & - & - & 1 & - & - \\
\hline Anorexia & 1 & - & - & 1.0 & - \\
\hline RIF pain & - & 3.51 & 1 & 0.5 & 2 \\
\hline Migratory pain to the RIF & 1 & - & - & 0.5 & 2 \\
\hline \multicolumn{6}{|l|}{ Duration of pain (hr) } \\
\hline$<48$ & - & - & - & 1.0 & - \\
\hline$\geq 48$ & - & 2.13 & - & 0.5 & - \\
\hline \multicolumn{6}{|l|}{ Signs } \\
\hline RIF tenderness & 2 & 11.41 & - & 1.0 & $3 / 1^{\text {a) }}$ \\
\hline Rebound tenderness & 1 & 4.25 & - & 1.0 & - \\
\hline Muscular defense/rebound & & 6.62 & & 2.0 & \\
\hline Mild & - & - & 1 & - & 2 \\
\hline Intermediate & - & - & 2 & - & 4 \\
\hline Strong & - & - & 3 & - & 4 \\
\hline \multicolumn{6}{|l|}{ Temperature $\left({ }^{\circ} \mathrm{C}\right)$} \\
\hline$\geq 37.3$ & 1 & - & - & $1.0^{b)}$ & - \\
\hline$\geq 38.5$ & - & - & 1 & - & - \\
\hline Rovsing's sign & - & - & - & 2.0 & - \\
\hline \multicolumn{6}{|l|}{ Clinical results } \\
\hline \multicolumn{6}{|l|}{ White blood cell count $\left(\times 10^{9} / \mathrm{L}\right)$} \\
\hline$\geq 10.0$ & 2 & 5.88 & 1 & $1.0^{6)}$ & - \\
\hline$\geq 7.2$ and $<10.9$ & - & - & - & - & 1 \\
\hline$\geq 10.9$ and $<14.0$ & - & - & - & - & 2 \\
\hline$\geq 14.0$ & - & - & - & - & 3 \\
\hline$\geq 15.0$ & - & - & 2 & - & - \\
\hline \multicolumn{6}{|l|}{ Polymorphonuclear leukocytes (\%) } \\
\hline$>75$ & 1 & - & - & - & - \\
\hline$\geq 70$ and $<85$ & - & - & 1 & - & - \\
\hline$\geq 85$ & - & - & 2 & - & - \\
\hline$\geq 62$ and $<75$ & - & - & - & - & 2 \\
\hline$\geq 75$ and $<83$ & - & - & - & - & 3 \\
\hline$\geq 83$ & - & - & - & - & 4 \\
\hline \multicolumn{6}{|l|}{ C-reactive protein concentration $(\mathrm{mg} / \mathrm{L})$} \\
\hline$\geq 10$ and $<50$ & - & - & 1 & - & - \\
\hline$\geq 50$ & - & - & 2 & - & - \\
\hline$\geq 4$ and $<11$ (<24-hour symptoms) & - & - & & - & 2 \\
\hline$\geq 11$ and $<25$ ( $<24$-hour symptoms $)$ & - & - & & - & 3 \\
\hline$\geq 25$ and $<83$ ( $<24$-hour symptoms) & - & - & & - & 5 \\
\hline$\geq 83$ (<24-hour symptoms) & - & - & & - & 1 \\
\hline$\geq 12$ and $<53$ ( $\geq 24$-hour symptoms) & - & - & & - & 2 \\
\hline$\geq 53$ and $<152$ ( $\geq 24$-hour symptoms) & - & - & & - & 2 \\
\hline$\geq 152$ ( $\geq 24$-hour symptoms) & - & - & & - & 1 \\
\hline Negative urinalysis & - & - & - & 1.0 & - \\
\hline Total or maximum score & 10 & 67.60 & 12 & 16.0 & 25 \\
\hline
\end{tabular}

The variables which were not included in each clinical scoring system were marked as '-'.

AIR, appendicitis inflammatory response; RIPASA, Raja Isteri Pengiran Anak Saleha Appendicitis; AAS, adult appendicitis score; RIF, right iliac fossa.

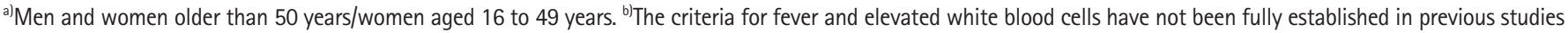
using the RIPASA score. Therefore, the score was evaluated using the criteria of our institution. 
Netherlands) with intravenous administration of a contrast agent, without the need for administration of oral contrast medium. The parameters used were as follows: anatomical range; scan from the diaphragm to the symphysis pubis with a collimation of 1.5 $\mathrm{mm}$; rotation time, 0.75 second; and pitch, 1.188. Reconstructions consisted of axial and coronal sections of 5 or $3 \mathrm{~mm}$. Tube voltage was $120 \mathrm{kVp}$, and tube current was 150 to $300 \mathrm{~mA}$. Enhanced CT of the entire abdomen was performed with a 60-second delay after the administration of a dose of $2 \mathrm{~mL} / \mathrm{kg}$ body weight of iohexol (Omnipaque 350, GE Healthcare, Princeton, NJ, USA) and iopamidol (Pamiray 370, Dongkook Pharmaceutical, Seoul, Korea), with an infusion rate of $4 \mathrm{~mL} / \mathrm{sec}$ through the antecubital vein.

\section{Image interpretation and definitions}

All CT examinations were analyzed by one experienced abdominal radiologist (10 years' experience). CT assessment of the appendix was based on a set of five criteria derived from previous studies: an appendix without intraluminal air with a distension of more than $6 \mathrm{~mm}$; periappendiceal fat stranding and infiltration; appendiceal wall enhancement or thickening; cecal apical wall thickening compared with the normal thickness of the wall of the ascending colon; and presence of extraluminal fluid collection or gas bubbles around the appendix. ${ }^{14-16}$ The diameter of the appendix was measured at the longest portion of the visible appendix on enhanced axial sections. In cases in which three or more of these findings were present, the patient was diagnosed with definitive appendicitis. In cases in which two findings were present, the patient was diagnosed with probable appendicitis. The presence of only one finding was considered as equivocal appendicitis. In cases in which the appendix was not observed, or the radiologist failed to trace the entire appendix, the radiologist concluded that the patient probably did not have appendicitis. ${ }^{17}$ The appendices that were filled with air and measured less than 9 $\mathrm{mm}$ in diameter were considered normal. ${ }^{18}$

\section{Statistical analysis}

All data were analyzed using IBM SPSS Statistics ver. 19 (IBM, Inc., Armonk, NY, USA) and MedCalc ver. 7.4 (MedCalc Software, Mariakerke, Belgium). Continuous data are expressed as means and standard deviation or medians (interquartile range) according to the results of normal distribution analysis. Categorical data and frequency distributions are shown as absolute values. A Mann-Whitney U-test and an independent t-test were conducted according to normal distribution analysis. Chi-square tests were conducted to compare data between the sexes.

The diagnostic performance of each of the scoring systems was compared by conducting a receiver operating characteristic (ROC) analysis using MedCalc ver. 7.4. The optimal cut-off value was calculated by maximizing the sum of sensitivity and specificity. The other two different cut-off values were obtained to score the probability of appendicitis as low, intermediate, or high. The low and high cut-off values included a point with high sensitivity (higher than 90\%) and specificity of at least 30\%, and a point with high specificity (higher than $85 \%$ ) and sensitivity of at least $20 \% .^{13}$ The cut-off value within these sensitivity and specificity limits was chosen where the diagnostic odds ratio (positive likelihood ratio/negative likelihood ratio) was the highest..$^{13} \mathrm{P}$-values smaller than 0.05 were considered statistically significant.

\section{RESULTS}

\section{General characteristics of the study population}

During the study period, 3,162 patients (older than 15 years) with suspected AA visited our medical institution and underwent intravenously enhanced abdominal CT to rule out AA. These patients received the following diagnoses: confirmed appendicitis $(n=1,025)$, probable appendicitis $(n=291)$, equivocal appendicitis ( $n=202)$, probably not appendicitis $(n=248)$, and normal appendix $(n=1,396)$. After excluding 13 patients with insufficient data on the clinical scoring systems from those with equivocal appendicitis $(n=202,6.4 \%), 189$ adult patients with suspected AA and equivocal CT findings were enrolled in this study (Fig. 1).

Of the 189 enrolled patients, AA was confirmed in 61 (32.3\%) patients using the aforementioned diagnostic criteria. There was no significant difference in age or sex ratio between the groups $(P=0.582, P=0.428)$. Negative appendectomy was defined as the absence of adult inflammation in the pathology reports. Seventyseven operations were performed, and the rate of negative appendectomy was $20.8 \%(16 / 77)$. The alternative diagnoses in patients subjected to surgery but not diagnosed with appendicitis were as follows: adult gastroenteritis $(n=6)$, pelvic inflammatory disease $(n=1)$, diverticulitis $(n=2)$, appendiceal mucocele $(n=1)$, mesenteric lymphadenopathy $(n=2)$, and non-specific findings $(n=4)$. Fifty-four patients $(70.1 \%)$ were referred to immediate surgery; of them, 41 (75.9\%) patients had AA. Twenty-three patients (29.9\%) were administered to antibiotic therapy before delayed surgery; of them, 20 (87.0\%) patients had AA (Fig. 1).

The final diagnosis in 112 patients who did not undergo operation were as follows: adult gastroenteritis $(n=42,37.5 \%)$, adult pyelonephritis $(n=3,2.7 \%)$, pelvic inflammatory disease $(n=18$, $16.1 \%)$, diverticulitis $(n=5,4.5 \%)$, epiploic appendagitis $(n=1$, $0.9 \%)$, mesenteric lymphadenopathy $(n=8,7.1 \%)$, terminal ileitis $(n=3,2.7 \%)$, pseudomembranous colitis $(n=1,0.9 \%)$, ureteral 


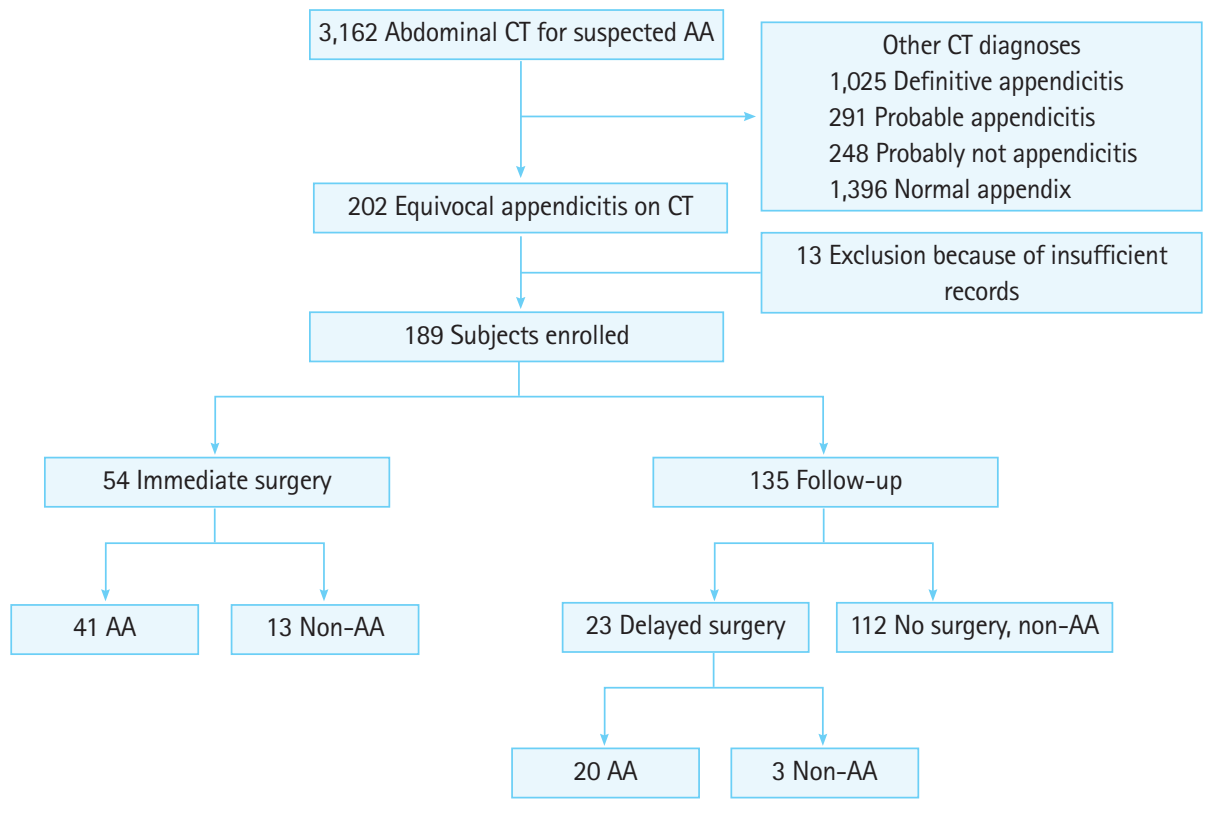

Fig. 1. Study design flowchart. CT, computed tomography; AA, adult appendicitis.

Table 2. Clinical scores and classification of the patients into three groups according to probability values

\begin{tabular}{|c|c|c|c|}
\hline & Appendicitis group & Non-appendicitis group & P-value \\
\hline Sex, male & $22(36.1)$ & $41(32.0)$ & 0.582 \\
\hline Age $(y r)$ & $31.0(22.0-43.0)$ & $35.0(24.5-43.5)$ & 0.428 \\
\hline \multicolumn{4}{|l|}{ Clinical score } \\
\hline Alvarado & $6(4-7)$ & $4(3-6)$ & $<0.001$ \\
\hline Eskelinen & $36.04(34.41-40.29)$ & $32.29(30.16-36.04)$ & $<0.001$ \\
\hline AIR & $5(3-5)$ & $3(2-5)$ & $<0.001$ \\
\hline RIPASA & $5.5(5.0-7.0)$ & $5.0(4.0-6.0)$ & 0.001 \\
\hline AAS & $11(9-14)$ & $8(5-11)$ & $<0.001$ \\
\hline \multicolumn{4}{|c|}{ Patient classification according to probabilities } \\
\hline Alvarado & & & 0.006 \\
\hline $\operatorname{Low}(\leq 2)$ & 2 & 19 & \\
\hline Intermediate $(2<$ score $\leq 7)$ & 50 & 103 & \\
\hline $\operatorname{High}(>7)$ & 9 & 6 & \\
\hline Eskelinen & & & 0.002 \\
\hline $\operatorname{Low}(\leq 28.03)$ & 0 & 3 & \\
\hline Intermediate $(28.03<$ score $\leq 40.29)$ & 54 & 124 & \\
\hline High $(>40.29)$ & 7 & 1 & \\
\hline AIR & & & 0.001 \\
\hline $\operatorname{Low}(\leq 1)$ & 3 & 24 & \\
\hline Intermediate $(1<$ score $\leq 6)$ & 51 & 102 & \\
\hline $\operatorname{High}(>6)$ & 7 & 2 & \\
\hline RIPASA & & & $<0.001$ \\
\hline $\operatorname{Low}(\leq 3.5)$ & 3 & 21 & \\
\hline Intermediate $(3.5<$ score $\leq 7.5)$ & 48 & 106 & \\
\hline $\operatorname{High}(>7.5)$ & 10 & 1 & \\
\hline AAS & & & $<0.001$ \\
\hline $\operatorname{Low}(\leq 5)$ & 3 & 33 & \\
\hline Intermediate $(5<$ score $\leq 15)$ & 50 & 90 & \\
\hline High $(>15)$ & 8 & 5 & \\
\hline
\end{tabular}

Values are presented as number (\%), median (interquartile range, $25 \%$ to $75 \%$ ), or number.

AIR, appendicitis inflammatory response; RIPASA, Raja Isteri Pengiran Anak Saleha Appendicitis; AAS, adult appendicitis score. 
stone $(n=2,1.8 \%)$, ovarian cyst rupture $(n=3,2.7 \%)$, and functional gastroenteritis $(n=26,23.2 \%)$.

\section{Clinical scores, ROC analysis, and diagnostic performance} of clinical scores

Table 2 shows the results of the median and interquartile range of each clinical score. The optimal cut-off values were higher than 5 using Alvarado score, 38.17 using Eskelinen score, 4 using AIR score, 5 using RIPASA score, and 8 using AAS score. Based on the chosen low and high cut-off values in the ROC analysis for each scoring system, the study participants were classified into three groups. The cut-off values, absolute and relative number of patients, and P-values are summarized in Table 2. Ten out of 11 (90.9\%) patients in the high probability group were diagnosed with AA using RIPASA score, indicating a high positive predictive value (90.9). In addition, only 3 out of 36 (8.3\%) patients in the low probability group using the AAS score were diagnosed with $A A$, indicating a high negative predictive value (91.7). There was no significant difference in the area under the curve for each clinical score (Alvarado, 0.698 [0.627 to 0.762]; Eskelinen, 0.710 [0.640 to 0.774]; AIR, 0.668 [0.596 to 0.734]; RIPASA, 0.653
Table 3. Sensitivity, specificity, positive predictive value, and negative predictive value of the clinical scores according to each cut-off value

\begin{tabular}{ccccc}
\hline Scores & Sensitivity (95\% Cl) & Specificity (95\% Cl) & PPV & NPV \\
\hline $\begin{array}{c}\text { Alvarado } \\
>2\end{array}$ & $96.7(88.6-99.5)$ & $14.8(9.2-22.2)$ & 35.1 & 90.5 \\
$>5$ & $55.7(42.4-68.5)$ & $74.2(65.7-81.5)$ & 50.7 & 77.9 \\
$\quad>7$ & $14.8(7.0-26.2)$ & $95.3(90.1-98.2)$ & 60.0 & 70.1 \\
Eskelinen & & & & \\
$>28.03$ & $100(94.1-100.0)$ & $2.3(0.5-6.7)$ & 32.8 & 100.0 \\
$>38.17$ & $45.9(33.1-59.1)$ & $89.1(82.3-93.9)$ & 66.7 & 77.6 \\
$>40.29$ & $11.5(4.8-22.2)$ & $99.2(95.7-99.9)$ & 87.5 & 70.2 \\
AIR & & & & \\
$>1$ & $95.1(86.3-98.9)$ & $18.8(12.4-26.6)$ & 35.8 & 88.9 \\
$>4$ & $52.5(39.3-65.4)$ & $72.7(64.1-80.2)$ & 47.8 & 76.2 \\
$>6$ & $11.5(4.8-22.2)$ & $98.4(94.5-99.8)$ & 77.8 & 70.0 \\
RIPASA & & & & \\
$>3.5$ & $95.1(86.3-98.9)$ & $16.4(10.5-24.0)$ & 35.2 & 87.5 \\
$>5$ & $62.3(49.0-74.4)$ & $58.6(49.6-67.2)$ & 41.8 & 76.5 \\
$>7.5$ & $16.4(8.2-28.1)$ & $99.2(95.7-99.9)$ & 90.9 & 71.3 \\
AAS & & & & \\
$>5$ & $95.1(86.3-98.9)$ & $25.8(18.5-34.3)$ & 37.9 & 91.7 \\
$>8$ & $82(70.0-90.6)$ & $53.9(44.9-62.7)$ & 45.9 & 86.3 \\
$>15$ & $13.1(5.9-24.2)$ & $96.1(91.1-98.7)$ & 61.5 & 70.0 \\
\hline
\end{tabular}

$\mathrm{Cl}$, confidence interval; PPV, positive predictive value; NPV, negative predictive value; AIR, appendicitis inflammatory response; RIPASA, Raja Isteri Pengiran Anak Saleha Appendicitis; AAS, adult appendicitis score.
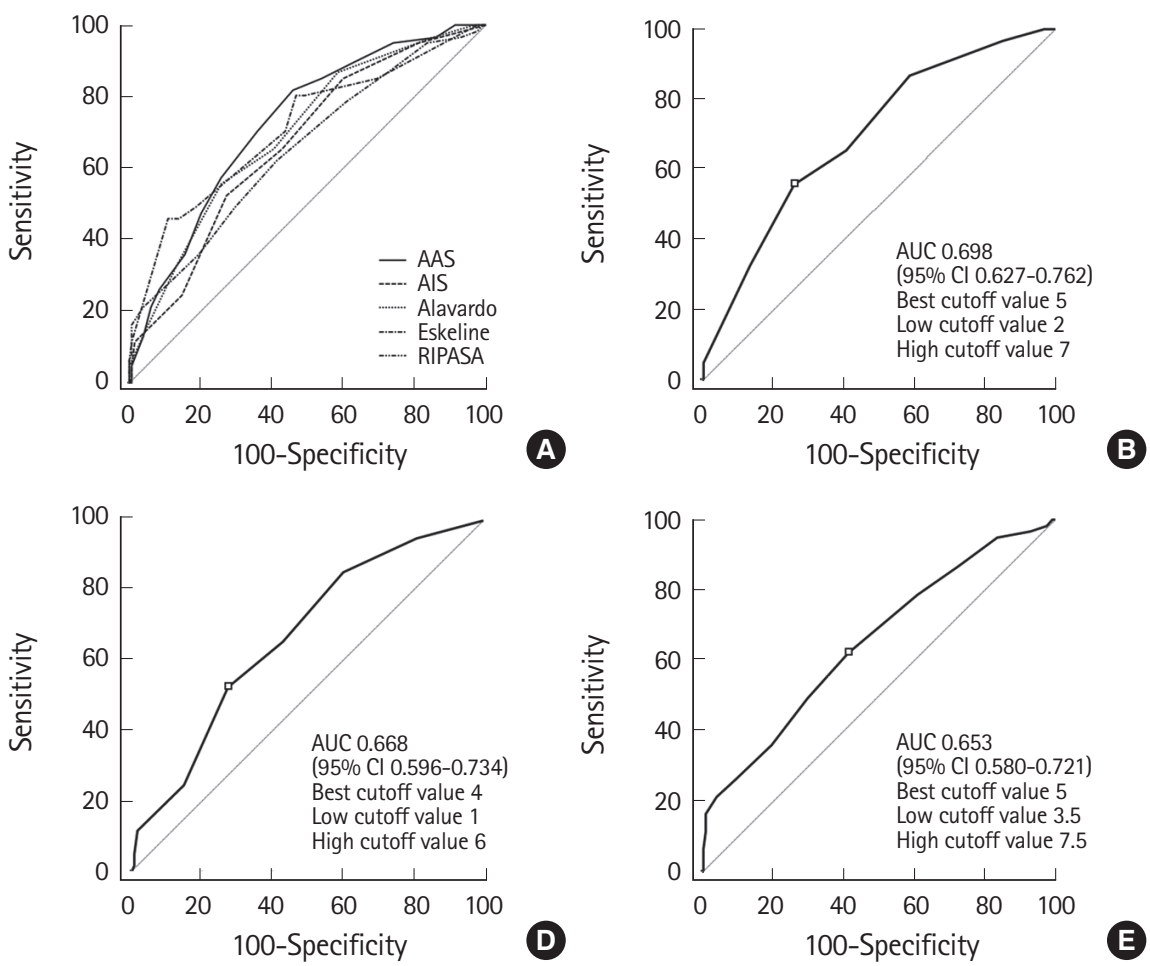

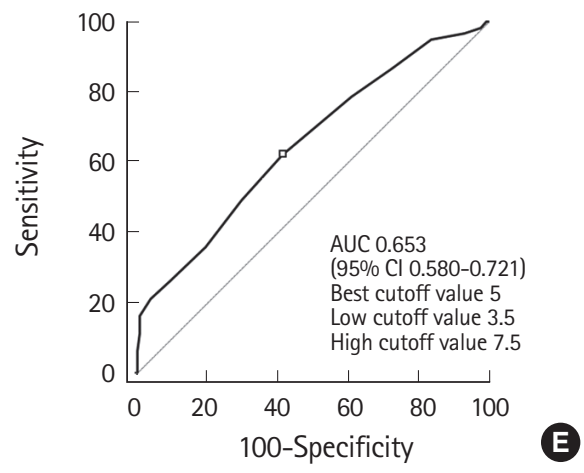

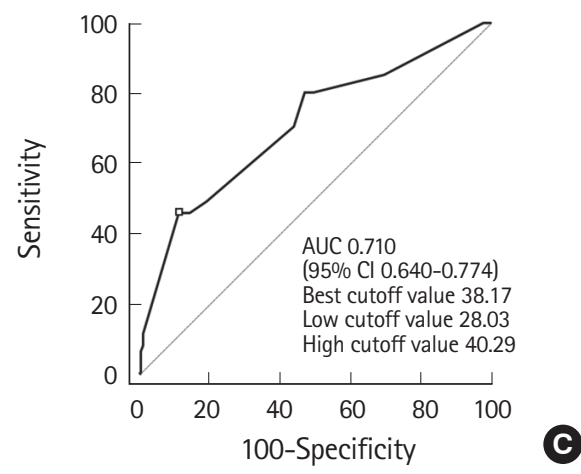

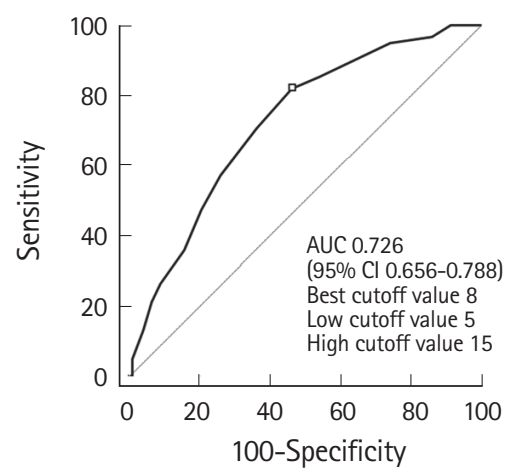

Fig. 2. Receiver operating characteristic (ROC) curve and area under the curve (AUC) of each clinical score for the diagnosis of adult appendicitis. (A) ROC of all scores, (B) ROC of Alvarado score, (C) ROC of Eskelinen score, (D) ROC of appendicitis inflammatory response (AIR), (E) ROC of Raja Isteri Pengiran Anak Saleha Appendicitis (RIPASA), and (F) ROC of adult appendicitis score (AAS).

There was no statistical difference between the scores by pairwise comparison of the ROC curves $(\mathrm{P}>0.05)$. P-values were calculated using MedCalc ver. 7.4 (MedCalc Software, Mariakerke, Belgium) to compare AAS with other scores. The square mark on each ROC curve indicates the best cutoff value for each clinical score. $\mathrm{Cl}$, confidence interval. 
[0.580 to 0.721 ]; AAS, 0.726 [0.656 to 0.788$]$ ) by pairwise comparison analysis between AAS and other scores. Fig. 2 and Table 3 summarize the results.

\section{DISCUSSION}

Patients with equivocal CT scans are a diagnostic challenge in the emergency department because the probability of appendicitis in this group is approximately $30 \% .^{8}$ Ultrasound re-evaluation and reassessment of $\mathrm{CT}$ images improve the accuracy of diagnosis of AA. ${ }^{19}$ In most institutions, ultrasound re-evaluation and reassessment of CT are not frequently used in the diagnosis of AA. Therefore, clinical scoring systems that are simple, non-invasive, and easy to use in routine clinical practice may provide useful information to clinicians. In this study, the diagnostic accuracy of clinical scoring systems was moderate (Alvarado, 0.698; Eskelinen, 0.710; AIR, 0.668; RIPASA, 0.653; AAS, 0.726). However, most patients presented with intermediate scores: 153 (81.0\%) patients using the Alvarado score, 178 (94.2\%) using the Eskelinen score, 153 (81.0\%) using the AIR score, 154 (81.5\%) using the RIPASA score, and 140 (74.1\%) using the AAS score. However, the most significant results were that 1) using AAS score, the low probability group $(\leq 5)$ showed a high negative predictive value (91.7) with potential to safely exclude the occurrence of $A A ; 2$ ) using RIPASA score, the high probability group (>7.5) showed a high positive predictive value (90.9) with potential to help diagnose AA. In addition, AAS and RIPASA scores could help in 47 out of $189(24.8 \%)$ patients with equivocal appendicitis to include or exclude the diagnosis of appendicitis.

The diagnostic accuracy of the clinical scoring systems was moderate probably because of the stage of appendicitis. ${ }^{20}$ The patients with $\mathrm{AA}$ with equivocal $\mathrm{CT}$ findings were in the early stage of appendicitis, and their symptoms might not be characteristic of AA. The cut-off values obtained in previous studies differed from those of the present study. Accordingly, previous studies found cut-off values of 4 to 7 for Alvarado score, 55 to 57 for Eskelinen score, 4 to 8 for AIR score, 5 to 7.5 for RIPASA score, and 11 to 18 for AAS score. ${ }^{9-13}$ The cut-off values in our study were lower than that found in previous studies probably because of the early stage of appendicitis in our patients.

The present study has several limitations. First, the retrospective design limits the diagnostic performance of the clinical scoring systems. In addition, it was impossible to use other scoring systems owing to the limited information in the medical records. Second, CT findings were interpreted by a single expert abdominal radiologist, and the inter-observer correlation was not determined. Therefore, selection bias for equivocal CT findings might have occurred. Moreover, standards or criteria of equivocal appendicitis are controversial. The criteria used in our study in cases of equivocal CT were established and used in a single institution; therefore, any generalized conclusions about these criteria could not be made, and additional large-scale studies are required to elucidate this issue. Third, 58 of 112 (51.8\%) patients in the nonappendicitis group were treated with antibiotics. The success rate of antibiotic therapy alone in uncomplicated appendicitis is $72.7 \% .^{20}$ Although the rates of equivocal CT findings and confirmed appendicitis in these patients were similar to those of previous studies, ${ }^{8}$ the true incidence of appendicitis in the patients with equivocal CT findings might have been underestimated in cases in which the disease was cured with antibiotics in any of the 58 patients. Therefore, the incidence of $A A$ in equivocal CT findings and the rate of negative appendectomy in our study could not be generalized. Furthermore, the study population was relatively small. Therefore, other large-scale, randomized, controlled studies are required to validate our results.

In conclusion, the overall diagnostic accuracy of the clinical scoring systems was moderate. Forty-seven of 189 patients $(24.8 \%)$ were accurately diagnosed with appendicitis or non-appendicitis using the AAS and RIPASA scores. Although it is difficult to make general conclusions because of study limitations (including the retrospective design and small sample size), the RIPASA score might be considered better to diagnose AA in the patients with equivocal CT findings and the AAS score might be considered a better criterion for the safe discharge of patients from the emergency department. Most patients presented with intermediate scores. The patients with equivocal CT findings might be considered as a third diagnostic group for AA.

\section{CONFLICT OF INTEREST}

No potential conflict of interest relevant to this article was reported.

\section{REFERENCES}

1. Andersson RE. Meta-analysis of the clinical and laboratory diagnosis of appendicitis. Br J Surg 2004;91:28-37.

2. Impellizzeri $P$, Centonze $A$, Antonuccio $P$, et al. Utility of a scoring system in the diagnosis of acute appendicitis in pediatric age: a retrospective study. Minerva Chir 2002;57:341-6.

3. Jones K, Pena AA, Dunn EL, Nadalo L, Mangram AJ. Are negative appendectomies still acceptable? Am J Surg 2004;188: 748-54.

4. Vadeboncoeur TF, Heister RR, Behling CA, Guss DA. Impact of 
helical computed tomography on the rate of negative appendicitis. Am J Emerg Med 2006;24:43-7.

5. Sun JS, Noh HW, Min YG, et al. Receiver operating characteristic analysis of the diagnostic performance of a computed tomographic examination and the Alvarado score for diagnosing acute appendicitis: emphasis on age and sex of the patients. J Comput Assist Tomogr 2008;32:386-91.

6. Levine $C D$, Aizenstein 0 , Lehavi 0 , Blachar A. Why we miss the diagnosis of appendicitis on abdominal CT: evaluation of imaging features of appendicitis incorrectly diagnosed on CT. AJR Am J Roentgenol 2005;184:855-9.

7. Webb EM, Wang Z, Coakley FV, Poder L, Westphalen AC, Yeh BM. The equivocal appendix at CT: prevalence in a control population. Emerg Radiol 2010;17:57-61.

8. Daly CP, Cohan RH, Francis IR, Caoili EM, Ellis JH, Nan B. Incidence of acute appendicitis in patients with equivocal CT findings. AJR Am J Roentgenol 2005;184:1813-20.

9. Alvarado A. A practical score for the early diagnosis of acute appendicitis. Ann Emerg Med 1986;15:557-64.

10. Andersson $M$, Andersson RE. The appendicitis inflammatory response score: a tool for the diagnosis of acute appendicitis that outperforms the Alvarado score. World J Surg 2008;32: 1843-9.

11. Chong CF, Thien A, Mackie AJ, et al. Comparison of RIPASA and Alvarado scores for the diagnosis of acute appendicitis. Singapore Med J 2011;52:340-5.

12. Erdem H, Cetinkunar S, Das K, et al. Alvarado, Eskelinen, Ohhmann and Raja Isteri Pengiran Anak Saleha Appendicitis scores for diagnosis of acute appendicitis. World J Gastroenterol 2013;19:9057-62.
13. Sammalkorpi HE, Mentula P, Leppaniemi A. A new adult appendicitis score improves diagnostic accuracy of acute appendicitis: a prospective study. BMC Gastroenterol 2014;14: 114.

14. Rao PM, Rhea JT, Novelline RA, et al. Helical CT technique for the diagnosis of appendicitis: prospective evaluation of a focused appendix CT examination. Radiology 1997;202:139-44.

15. Choi $D$, Park $H$, Lee $Y R$, et al. The most useful findings for diagnosing acute appendicitis on contrast-enhanced helical CT. Acta Radiol 2003;44:574-82.

16. Pinto Leite N, Pereira JM, Cunha R, Pinto P, Sirlin C. CT evaluation of appendicitis and its complications: imaging techniques and key diagnostic findings. AJR Am J Roentgenol 2005;185:406-17.

17. Nikolaidis $P$, Hwang CM, Miller FH, Papanicolaou N. The nonvisualized appendix: incidence of acute appendicitis when secondary inflammatory changes are absent. AJR Am J Roentgenol 2004;183:889-92.

18. Tamburrini S, Brunetti A, Brown M, Sirlin CB, Casola G. CT appearance of the normal appendix in adults. Eur Radiol 2005; 15:2096-103.

19. Sim JY, Kim HJ, Yeon JW, et al. Added value of ultrasound reevaluation for patients with equivocal CT findings of acute appendicitis: a preliminary study. Eur Radiol 2013;23:188290.

20. Salminen P, Paajanen $H_{\text {, Rautio }} T_{\text {, et al. Antibiotic therapy vs }}$ appendectomy for treatment of uncomplicated acute appendicitis: the APPAC randomized clinical trial. JAMA 2015;313: 2340-8 